\title{
Article \\ Genus-Wide Characterization of Nuclear Mitochondrial DNAs in Bumblebee (Hymenoptera: Apidae) Genomes
}

\author{
Lele Ding, Huiling Sang and Cheng Sun * \\ Institute of Apiculture Research, Chinese Academy of Agricultural Sciences, Beijing 100093, China; \\ dingleledinglele@163.com (L.D.); sanghuiling2020@163.com (H.S.) \\ * Correspondence: suncheng@caas.cn
}

Simple Summary: The DNA of mitochondria can be transferred into the nucleus and form nuclear mitochondrial DNAs (NUMTs). In this study, we identified and characterized NUMTs in genus-wide bumblebee species. The number of NUMTs in bumblebee is much lower than those in its closely related taxon, honeybee. The insertion sites of NUMTs in bumblebee are not random, with AT-rich regions harboring more NUMTs. In addition, NUMTs derived from the mitochondrial COX1 gene are most abundant in the nuclear genome. While the majority of NUMTs seem unfunctional in the bumblebee, some NUMTs show functional clues, which could fuse with their flanking sequences to form novel proteins. Our results shed light on the molecular features of NUMTs and uncover their contribution to genome innovation in the bumblebee.

Citation: Ding, L.; Sang, H.; Sun, C. Genus-Wide Characterization of Nuclear Mitochondrial DNAs in Bumblebee (Hymenoptera: Apidae) Genomes. Insects 2021, 12, 963. https: / / doi.org/10.3390/ insects12110963

Academic Editors: Monica Poelchau, Surya Saha and Brian Johnson

Received: 18 May 2021

Accepted: 13 October 2021

Published: 23 October 2021

Publisher's Note: MDPI stays neutral with regard to jurisdictional claims in published maps and institutional affiliations.

Copyright: (c) 2021 by the authors. Licensee MDPI, Basel, Switzerland. This article is an open access article distributed under the terms and conditions of the Creative Commons Attribution (CC BY) license (https:// creativecommons.org/licenses/by/ $4.0 /)$.

\begin{abstract}
In eukaryotes, DNA of mitochondria is transferred into the nucleus and forms nuclear mitochondrial DNAs (NUMTs). Taking advantage of the abundant genomic resources for bumblebees, in this study, we de novo generated mitochondrial genomes (mitogenomes) for 11 bumblebee species. Then, we identified and characterized NUMTs in genus-wide bumblebee species. The number of identified NUMTs varies across those species, with numbers ranging from 32 to 72, and nuclear genome size is not positively related to NUMT number. The insertion sites of NUMTs in the nuclear genome are not random, with AT-rich regions harboring more NUMTs. In addition, our results suggest that NUMTs derived from the mitochondrial COX1 gene are most abundant in the bumblebee nuclear genome. Although the majority of NUMTs are found within intergenic regions, some NUMTs do reside within genic regions. Transcripts that contain both the NUMT sequence and its flanking non-NUMT sequences could be found in the bumblebee transcriptome, suggesting a potential domestication of NUMTs in the bumblebee. Taken together, our results shed light on the molecular features of NUMTs in the bumblebee and uncover their contribution to genome innovation.
\end{abstract}

Keywords: bumblebee; mitogenome; NUMT; genome innovation

\section{Introduction}

In eukaryotes, the DNA of mitochondria can be transferred to the nuclear genome and form so-called nuclear mitochondrial DNAs (NUMTs) [1-6]. NUMTs are inserted into the nuclear genome during the repair of double-strand breaks (DSBs) by the non-homologous end-joining repair (NHEJ) pathway [4,7]. The number of NUMTs varies across species, and broadly, three reasons could underlie such variation: (1) the frequency of DSBs is different across species; (2) the frequency of DSBs repaired by NHEJ vary between species; and (3) the rate of NUMT loss is different across species [3,5,8]. After integration into the nuclear genome, NUMTs are believed to be mainly nonfunctional sequences, which could also disrupt nuclear functional genes (occasionally causing human genetic disease) or contribute beneficially to gene evolution [9-11]. The presence of NUMTs could be problematic when using mitochondrial DNA (mtDNA) as molecular markers in evolutionary studies; if mtDNA is amplified by PCRs using common methodology, NUMTs could be co-amplified, which will mislead downstream phylogenetic analysis [12]. 
Bumblebees (Hymenoptera: Apidae) are a group of insects that comprise the genus Bombus, with 250 extant species classified into 15 subgenera [13-15]. They are important pollinators for both wild flowers and crops [16,17]. Identification and characterization of NUMTs in bumblebees will not only uncover their molecular features (i.e., abundance, diversity, and evolution) and thus provide a general idea on to what extent NUMTs will affect phylogenetic analysis when using mtDNA as molecular markers in the bumblebee but will also shed light on the contribution of NUMTs to bumblebee gene and genome evolution. However, although the presence of NUMTs in the bumblebee has been implicated in a previous study [18], no detailed, genome-wide analysis has yet been conducted. Taking advantage of the genus-wide bumblebee genomic resources $[19,20]$, we performed a systematic examination of NUMTs in bumblebees to infer their abundance, origin, and evolution. Our study sheds light on the molecular features of NUMTs in bumblebee, as well as their contribution to genome innovation.

\section{Materials and Methods}

For bumblebee species that already have a reported mitogenome, their mitogenome sequences were downloaded from GenBank. For species that do not yet have a mitogenome, their Illumina shotgun reads for whole-genome sequencing were downloaded from NCBI Sequence Read Archive (SRA) (https: / / www.ncbi.nlm.nih.gov / sra (accessed on 28 August 2020)). The accession numbers for mitogenome sequences and Illumina shotgun reads are summarized in Table 1 . The genomic sequences and genome annotation of B. terrestris were downloaded from GenBank (assembly accession: GCF_000214255.1). The genome assemblies and annotations for all the other bumblebees were downloaded from the National Genomics Data Center (https: / /ngdc.cncb.ac.cn (accessed on 28 August 2020)), under BioProject number PRJCA002292.

Table 1. Sequences used in this study and their accession numbers.

\begin{tabular}{cccc}
\hline Species & Genomic Shotgun Reads & Published Mitogenomes & RNA-Seq \\
\hline B. breviceps & & MF478986 & SRR12527963 \\
B. confusus & SRR12527987 & MF995069 & SRR12527965 \\
B. consobrinus & SRR12528003 & & \\
B. cullumanus & SRR12527947 & & SRR12527962 \\
B. difficillimus & SRR12527992 & SRR12527929 \\
B. haemorrhoidalis & SR870926 & SRR12527935 \\
B. ignitus & SRR2527970 & & SRR12527964 \\
B. opulentus & SRR12528031 & & SRR12527936 \\
B. picipes & SRR12527998 & & \\
B. polaris & & MH998260 & SRR12527934 \\
B. pyrosoma & SRR12527981 & & SRR12527966 \\
B. sibiricus & SRR12528009 & & \\
B. soroeensis & SRR12528014 & & SRR12527932 \\
B. superbus & SRR12528020 & & SRR12527933 \\
B. turneri & & MK252702 & \\
B. waltoni & & KT368150 & \\
B. terrestris & & & \\
\hline
\end{tabular}

To generate mitogenome sequences, the downloaded Illumina shotgun reads of each species were assembled by NOVOPlasty v2.6.3 [21], MitoZ v2.4-alpha [22] and GetOrganelle v1.7.1 [23] software. When running NOVOPlasty, we used the B. terrestris COX1 gene as seed; when running MitoZ, parameters "-genetic_code 5 -clade Arthropoda run_mode 2 -filter_taxa_method 1 -requiring_taxa 'Arthropoda'" were applied; when running GetOrganelle, parameters "-R 10 -k 21,45,65,85,105 -F animal_mt” were used; all other parameters were set as default. The resulting mitogenomes were analyzed by using the MITOS web server [24]. Manual merging assisted by NCBI-BLAST2 was performed when one software generated more than one contigs and none of them contain all of 
the 13 mitochondrial protein-coding genes. The most complete mitogenomes (containing the most protein-coding and RNA genes-if the gene numbers were the same, then the longest sequence) obtained from the three assemblers were kept for each species for downstream analysis.

Bumblebee genomes are rich in AT [20]. To minimize false positives, NUMTs were identified by using mitogenome sequences as queries to perform BLASTn (-task blastn) against their respective nuclear genome sequences with an e-value cutoff of $6 \times 10^{-14}$, a strict threshold that has previously been applied in NUMT analysis in the honeybee genome [25]. Identified NUMTs that are located at the ends of each scaffold were removed from downstream analysis.

The coordinates of NUMTs were used to calculate their lengths, which were also compared with the coordinates of protein-coding genes of the same genome to infer the relative positions of NUMTs to its nearest genes. Then $100 \mathrm{bp}$ of upstream and downstream sequences of NUMTs in each genome were extracted, which were then analyzed by Genetyx7 software (GENETYX CORPORATION) to determine their AT content.

The correlation between NUMT number and mitogenome length, or nuclear genome length, was calculated by "cor.test ()" function in R (http:/ / www.R-project.org/ (accessed on 28 August 2020)).

For bumblebee species that have available RNA-seq data (Table 1) [19], shotgun reads were assembled using Trinity [26] with default parameters. For B. terrestris, whose genome has been intensively annotated, we used its curated transcripts directly (assembly accession: GCF_000214255.1). To identity chimeric transcripts that contain both NUMT sequence and flanking non-NUMT sequences, which suggests the potential domestication of NUMT (serving a novel function in the nuclear genome), the sequences of NUMT, along with its $60 \mathrm{bp}$ of flanking sequences, were extracted and used as queries for BLASTn against the assembled transcriptome sequence of each species, with an e-value cutoff of $1 \times 10^{-10}$ and a sequence identity cutoff of 0.98 . Transcripts that contain an NUMT sequence and at least $50 \mathrm{bp}$ of its flanking non-NUMT sequences were eligible chimeras.

\section{Results}

\subsection{Genus-Wide Mitogenome Resources for Bumblebees}

In this study, we de novo generated mitogenomes for 11 bumblebee species, whose mitogenome sizes range from 15,029 to $29,809 \mathrm{bp}$, with an average length of $17,839 \mathrm{bp}$ (Table 2). These mitogenomes are complete or almost complete: all of them have 13 protein-coding genes, and most of them have two rRNA genes and 22 tRNA genes (Table 2). Notably, B. sibiricus, which has the largest nuclear genome size across genus-wide species, also has the largest mitogenome size (Table 2). Coupled with previously reported bumblebee mitogenomes [27-31], we obtained mitogenomes for species spanning all of the 15 Bombus subgenera.

Table 2. Statistics for the 11 newly generated mitogenome sequences.

\begin{tabular}{ccccc}
\hline Species & $\begin{array}{c}\text { Total Length } \\
\mathbf{( b p )}\end{array}$ & $\begin{array}{c}\text { Protein-Coding } \\
\text { Gene }\end{array}$ & rRNA Gene & tRNA Gene \\
\hline B. confusus & 19,550 & 13 & 2 & 19 \\
B. cullumanus & 16,418 & 13 & 2 & 20 \\
B. difficillimus & 19,401 & 13 & 2 & 22 \\
B. haemorrhoidalis & 15,358 & 13 & 2 & 22 \\
B. opulentus & 17,293 & 13 & 1 & 21 \\
B. picipes & 18,381 & 13 & 2 & 22 \\
B. polaris & 16,785 & 13 & 2 & 22 \\
B. sibiricus & 29,809 & 13 & 2 & 22 \\
B. soroeensis & 17,138 & 13 & 2 & 19 \\
B. superbus & 15,792 & 13 & 2 & \\
B. turneri & 15,029 & 13 & & \\
\hline
\end{tabular}




\subsection{Molecular Features of NUMTs in Bumblebee Nuclear Genomes}

A total of 17 species were searched for NUMTs, and the results are summarized in Figure 1. From the results, we could see that NUMTs vary in number across species, ranging from 32 to 72 . The NUMT counts in the bumblebee are much lower than those in its closely related taxon honeybee, where more than 1000 NUMTs could be identified [25,32]. Regarding sequence length, NUMTs only comprised a tiny portion, ranging from $0.0030 \%$ to $0.0104 \%$ for each nuclear genome (Figure 1 ).

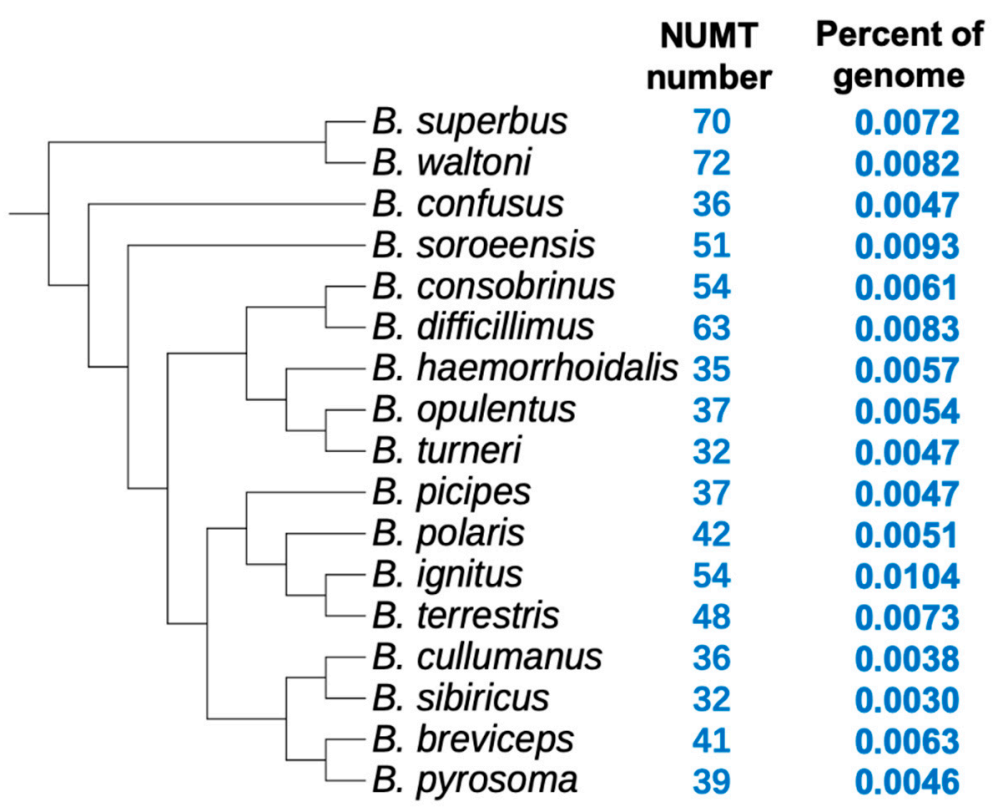

Figure 1. NUMTs identified in each of the 17 bumblebee species. The phylogeny was deduced from a previous study [19].

The length distribution of the NUMTs is summarized in Figure 2, from which we can observe that, while the majority of bumblebee NUMTs are less than $400 \mathrm{bp}$ in length, NUMTs longer than 2000 bp could also be found in some species.

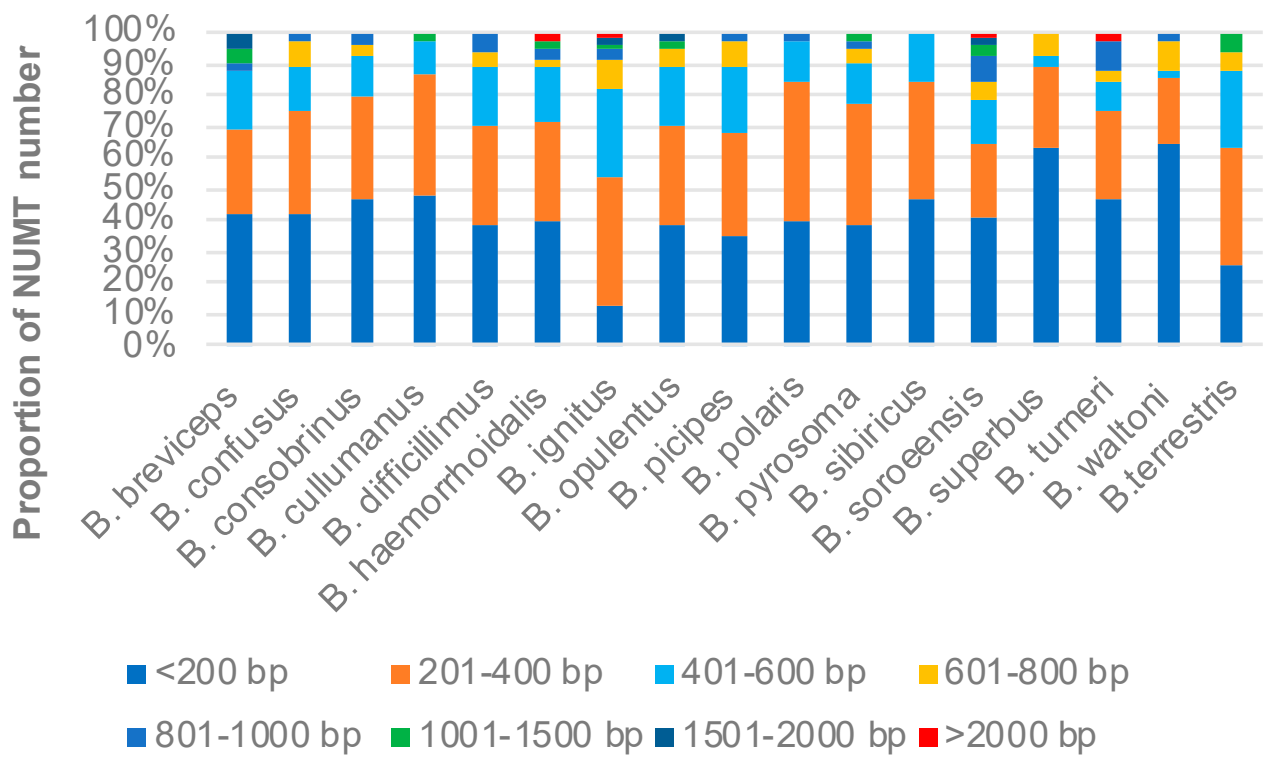

Figure 2. Length distribution of NUMTs in bumblebee. 
To understand factors that contribute to the variable number of NUMTs in different bumblebee species, firstly, we investigated the relationship between NUMT numbers and the length of mitogenomes. The results suggested that the variation of NUMT number is not correlated with mitogenome length $\left(R^{2}=0.0498 ; p\right.$-value $\left.=0.3891\right)$, ruling out the possibility that the completeness of mitogenome may underlie such variation. Then, we correlated NUMT number with nuclear genome size, and, unexpectedly, we identified a negative correlation between them $\left(R^{2}=0.3133\right.$; $p$-value $=0.02188$; Figure 3$)$. When we related the nuclear genome size with the total sequence length of NUMTs, no statistically significant correlation was identified ( $p$-value $>0.05)$.

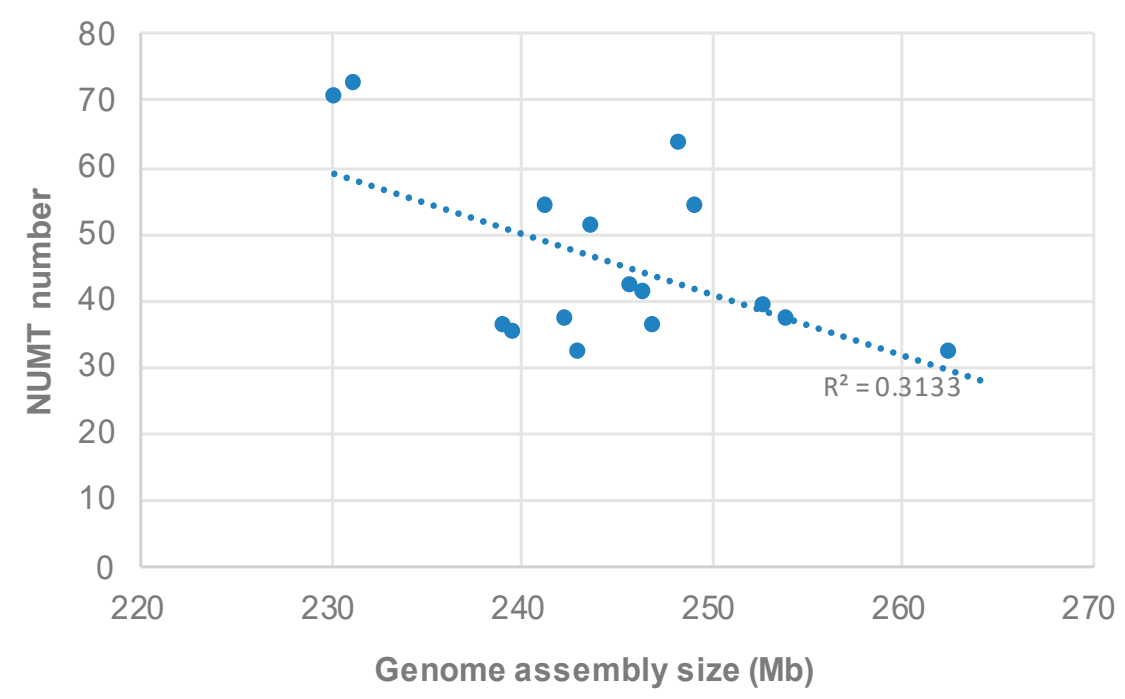

Figure 3. Relationship between NUMT number and nuclear genome size. Genome assembly sizes are from previous reports [19].

To understand the origin of NUMTs, we tracked NUMTs back to their respective mitogenomes. The numbers of NUMTs that were derived from each of the 13 mitochondrial coding genes were summarized for each species, and the results indicated that, in all of the 17 species, COX1-derived NUMTs are most frequently present in the nuclear genome (Figure 4). Our finding is similar to those in reports regarding the honey bee [25,32] and yeast [33].

To understand the sequence preference of NUMT insertion, we extracted $100 \mathrm{bp}$ of flanking sequences of NUMTs and investigated their sequence composition. Our results suggested that, in all of the 17 species, the AT contents of NUMT flanking sequences as a whole, upstream flanking sequences, and downstream flanking sequences are all higher than those of the whole nuclear genome (Figure 5), indicating a preference of the AT-rich sequence for NUMT insertion in the bumblebee nuclear genome. The preference of NUMTs for AT-rich regions has also been reported in fig wasp genomes [34]. Notably, the AT content of the NUMT upstream flanking sequence is consistently higher than that of the downstream flanking sequence in all of the 17 species (Figure 5). 


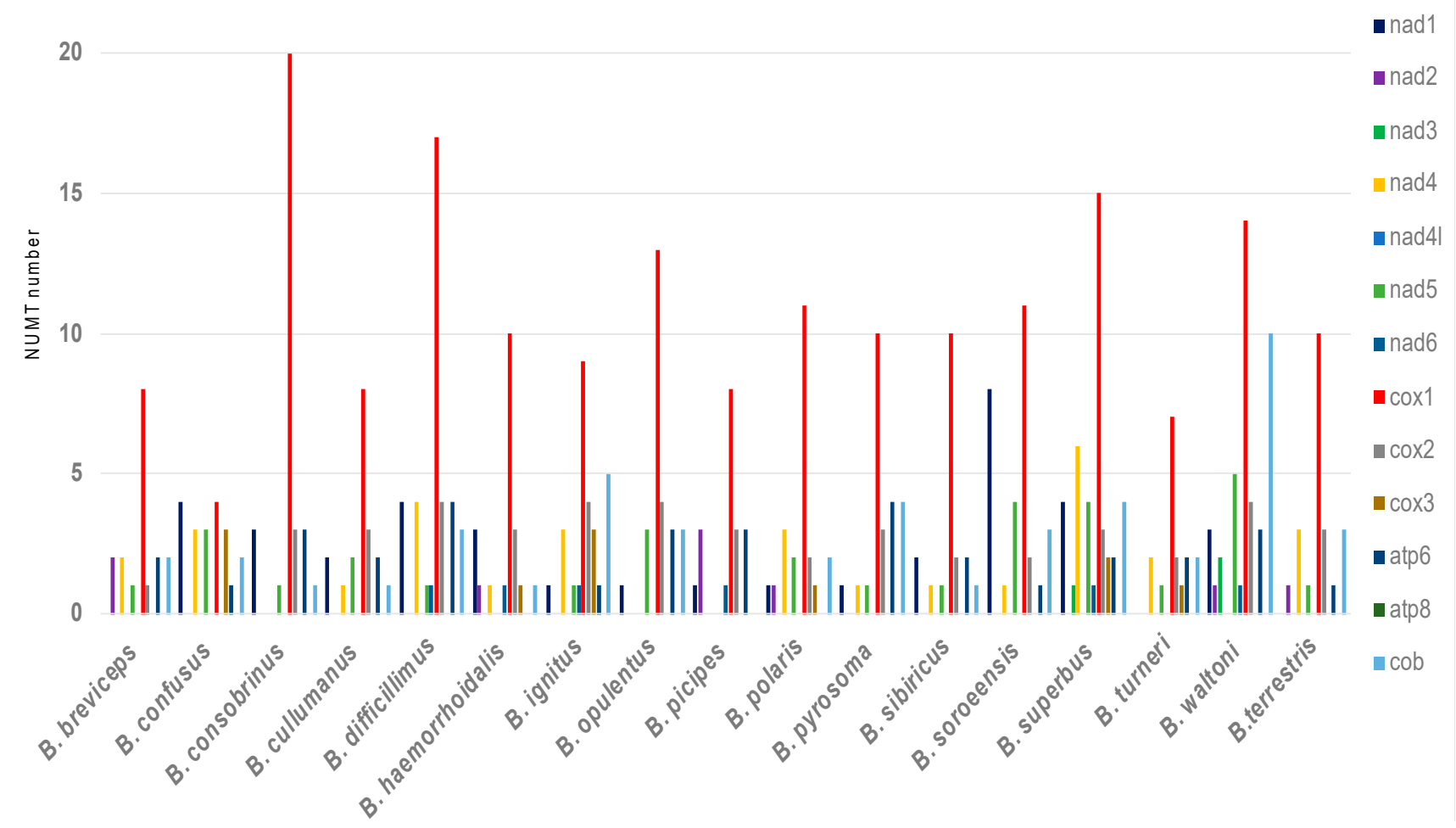

Figure 4. Number of NUMTs derived from each of the 13 mitochondrial protein-coding genes.

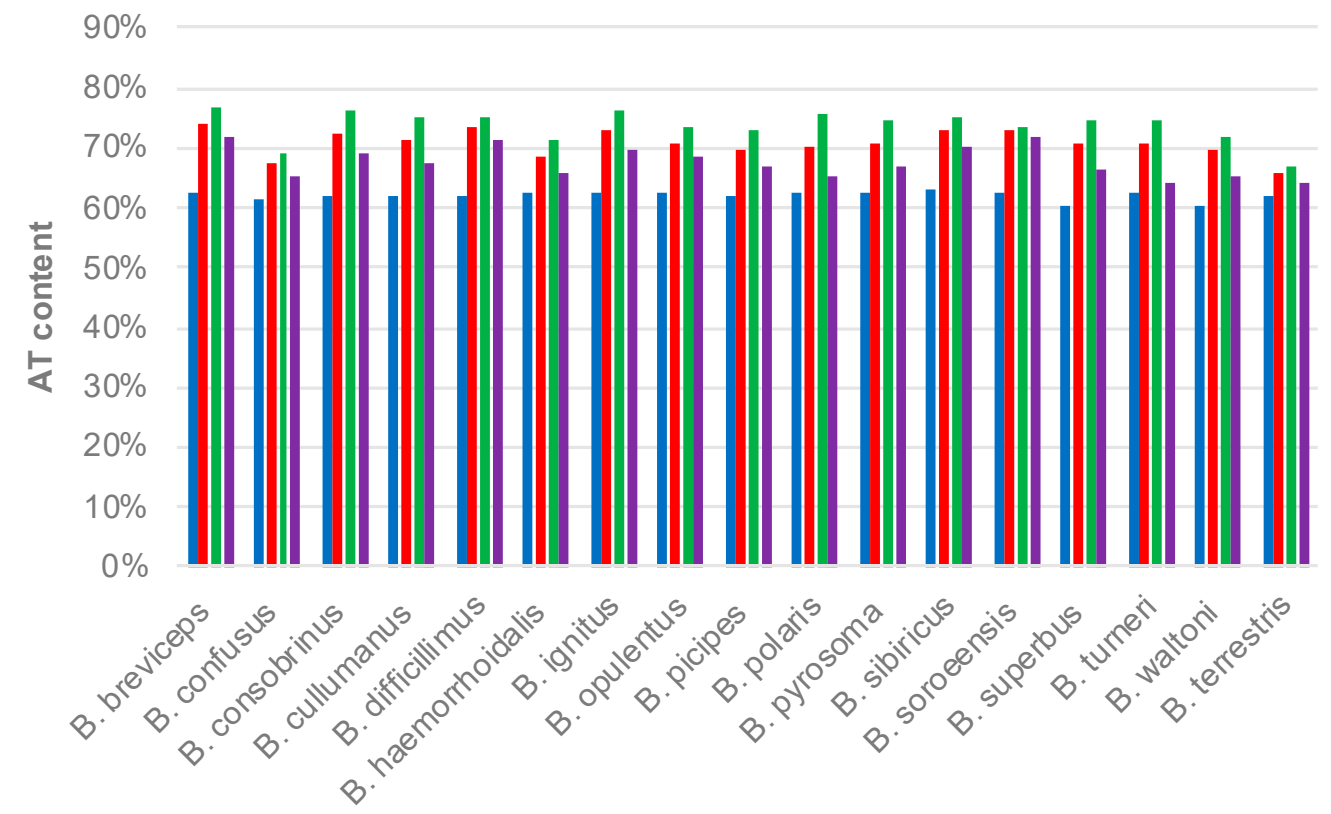

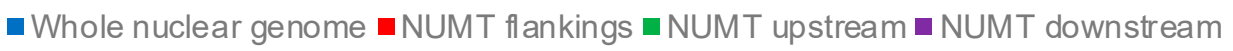

Figure 5. AT contents of the NUMT flanking sequences and whole nuclear genome.

\subsection{Contribution of NUMTs to Bumblebee Genome Innovation}

To understand if NUMTs could contribute to nuclear genome innovation in the bumblebee, the distribution of NUMTs in relation to nuclear protein-coding genes was analyzed. The results indicated that, in all of the 17 species, the majority of NUMTs were located within the intergenic regions (Figure 6). However, NUMTs, with numbers varying from 5 to 20 across species, were found within genic regions (exons and introns) or their $2 \mathrm{~Kb}$ 
of flanking (Figure 6). Considering their proximity to protein-coding genes, such NUMTs have the possibility of being domesticated in the nuclear genome.

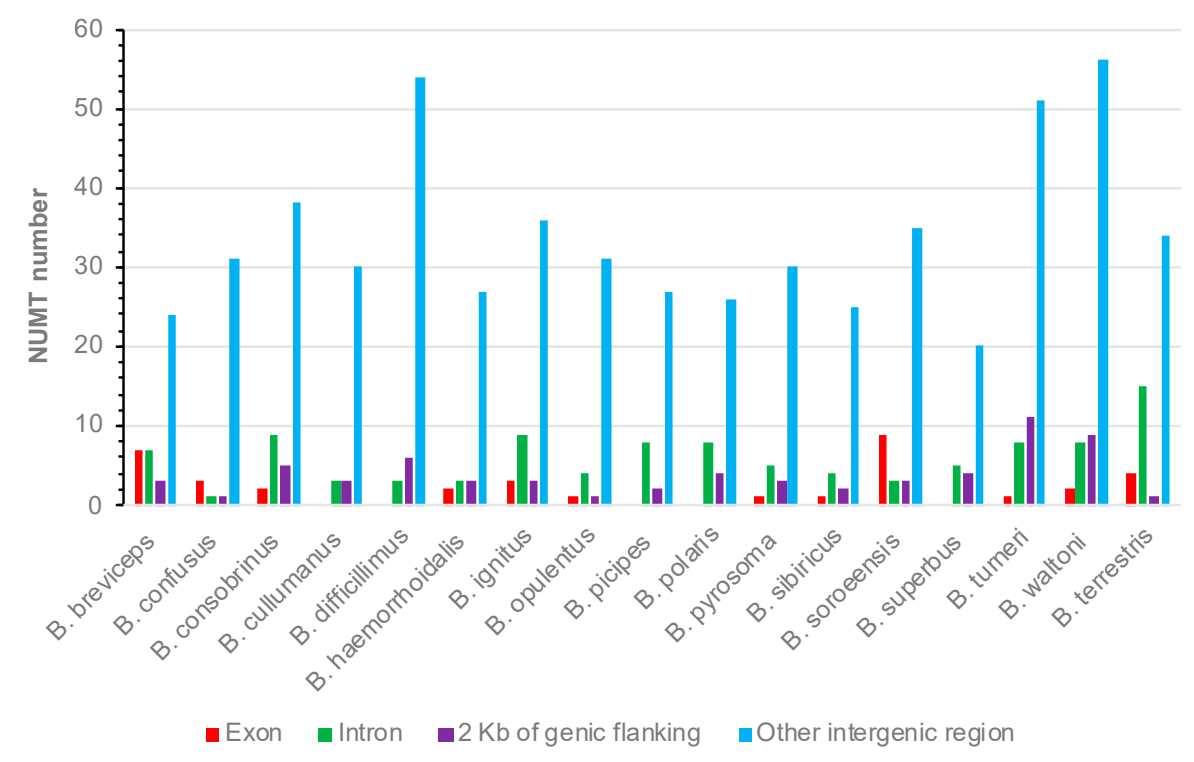

Figure 6. Distribution of NUMTs in relation to their nearest nuclear protein-coding genes.

To identify functional clues for those NUMTs, we identified NUMTs that fused with their flanking non-NUMT sequences and formed chimeras, for which we could find transcript evidence. Transcripts that contain the NUMT sequence and at least $50 \mathrm{bp}$ of its flanking non-NUMT sequences could be found in all of the 13 species, for which we have transcriptome data, with numbers ranging from 5 to 18 (Table 3). Here, we show one such example: in the $B$. terrestris gene mucin-5AC, one NUMT fused with its flanking sequences and formed a new functional exon (Figure 7). Therefore, our results suggest that some NUMTs might have been domesticated in the nuclear genome and contribute to nuclear genome innovation in the bumblebee.

Table 3. Summary of the fusion of NUMTs with their flanking sequences.

\begin{tabular}{cc}
\hline Species & Number of NUMTs Fused with Their Flanking Sequences \\
\hline B. breviceps & 18 \\
B. consobrinus & 8 \\
B. difficillimus & 9 \\
B. haemorrhoidalis & 9 \\
B. ignitus & 7 \\
B. opulentus & 7 \\
B. picipes & 8 \\
B. pyrosoma & 5 \\
B. sibiricus & 7 \\
B. superbus & 9 \\
B. turneri & 6 \\
B. waltoni & 12 \\
B. terrestris & 6 \\
\hline
\end{tabular}




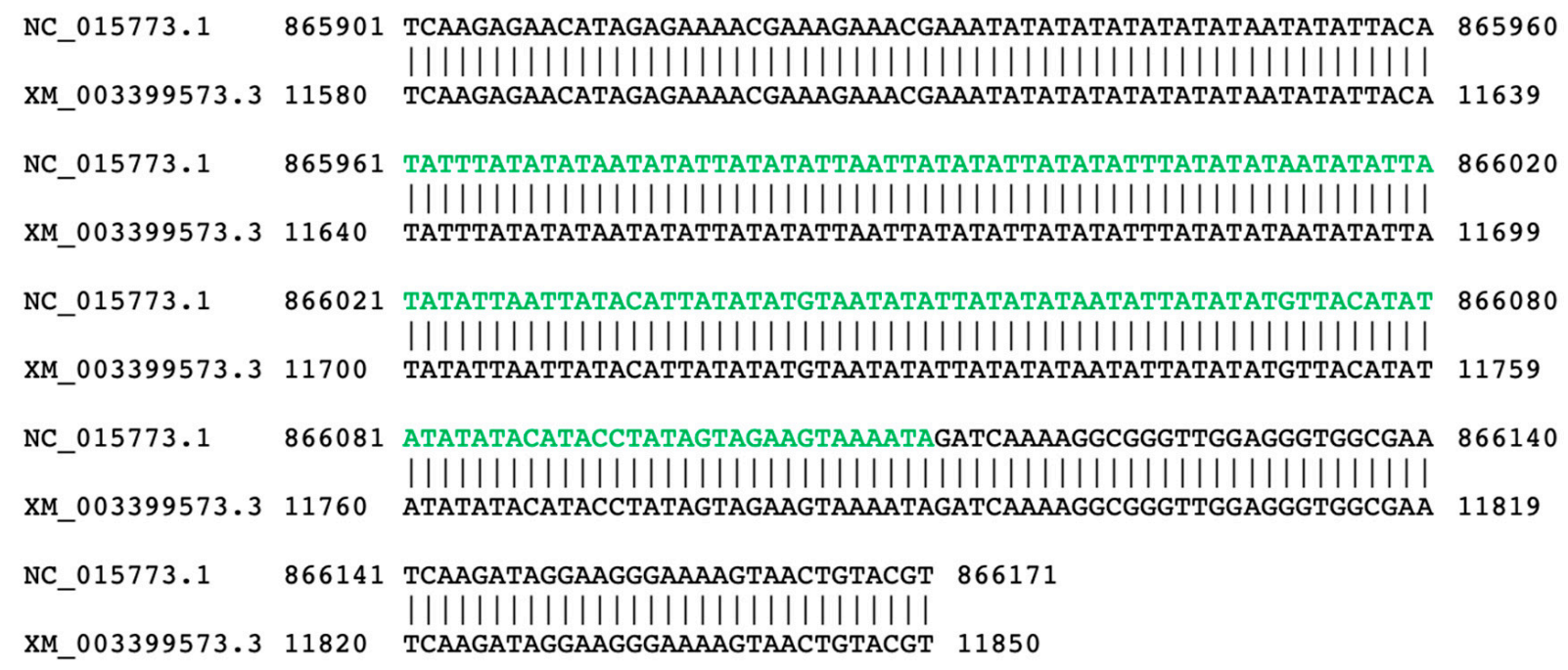

Figure 7. Domestication of one NUMT in bumblebee. Alignment between one genomic region of B. terrestris that harbors NUMT and one transcript that could support the transcription of NUMT. Characters in green indicate NUMT sequence. NC_015773.1 and XM_003399573.3 indicate the NCBI accession numbers for genomic sequence and transcript, respectively, with numbers flanking pairwise alignments indicating coordinates in each respective sequence. Based on the annotation report from NCBI, transcript XM_003399573.3 could be 100\% covered by RNA-seq alignments.

\section{Discussion}

In this study, using publicly available genomic resources and three popular software packages, we de novo generated mitogenome sequences for 11 bumblebee species (Table 2). To this end, we obtained mitogenomes for species spanning all of the 15 Bombus subgenera, which will facilitate studies on the evolution of mitogenomes, as well as the interaction between mitogenome and nuclear genome, in the bumblebee.

Some molecular features of NUMTs are conserved across genus-wide bumblebee species. For example, NUMTs derived from the mitochondrial COX1 gene are most abundant in all of the 17 analyzed bumblebee nuclear genomes (Figure 4). In the honeybee, the closely related taxa of the bumblebee, similar findings have been reported, which might be explained by the faster migration rate of this mtDNA region to the nucleus than that of the other mtDNA regions [25]. Our results also indicated that NUMTs are preferably present in AT-rich regions of the nuclear genome in all of the 17 focal species, and the AT content of NUMT upstream sequences is consistently higher than that of downstream sequences (Figure 5). The preference of AT-rich regions for bumblebee NUMTs is similar to the finding reported for humans, but no such preference has been identified in the honeybee [10,32].

The number of NUMTs considerably varies across the 17 analyzed species (Figure 1). Many factors have been proposed to affect the abundance of NUMTs, and when different sample sizes are used, different conclusions are obtained $[3,5,8]$. Comparative analysis of NUMTs in multiple closely related species, which could narrow down the genetic background difference between species, may provide new insights into factors underlying the variation of NUMT abundance. As NUMTs are integrated passively into the nuclear genome during the repair process of double-strand breaks (DSBs), we could expect that the more DSBs in one nuclear genome, the more NUMT insertions [2-4,7]. In yeast, the number of DSBs proportionally increased as the amount of nuclear DNA increased [35]; thus, if this trend is a general rule, then larger genomes will have more DSBs and, therefore, will have more NUMTs [3]. However, when we correlated nuclear genome sizes with NUMT counts across bumblebee species, we did not find such a positive correlation (instead, a negative correlation was observed) (Figure 3). In addition, when relating the nuclear genome size with the total sequence length of NUMTs, no statistically significant correlation was identified ( $p$-value $>0.05)$. Therefore, our results suggested that larger nuclear genomes, which should have more DSBs, do not necessarily harbor more NUMTs in the bumblebee. 
Future studies could investigate if the rate of NUMT loss or the frequency of DSBs repaired by NHEJ is different across bumblebee species, as these two factors could also contribute to the variable number of NUMTs across species $[3,5,8]$.

NUMTs are believed to be mainly nonfunctional sequences and could also cause disease or beneficially contribute to gene evolution [9-11]. In this study, we found that, while the majority of NUMTs were located within intergenic regions, there were NUMTs that reside within genic regions (Figure 6). In addition, we could identify transcripts that contain both NUMTs and their immediate flanking sequences, with numbers ranging from 5 to 18 across species, indicating that these NUMTs might have been domesticated in the nuclear genome to form functional exon sequences (Table 3; Figure 7). Here we used $50 \mathrm{bp}$ of flanking as a threshold to observe as many potential domestication events as possible, but such criteria may contain false positives, as chimeric RNA-seq reads could be generated during Illumina library preparation/sequencing. Taken together, our results in this study suggest that—other than 'junk' sequences—NUMTs could contribute to nuclear genome innovation in the bumblebee, which invites further experimental validation.

\section{Conclusions}

In this study, we performed comparative analysis of NUMTs across genus-wide bumblebee genomes. Our results indicated that NUMT number varies across bumblebee species, but nuclear genome size is not positively related to NUMT number. NUMTs have a preference of AT-rich regions in bumblebee nuclear genome, and NUMTs derived from the mitochondrial COX1 gene are most abundant. Although the majority of NUMTs seem unfunctional, we identified evidence that some NUMTs should have been domesticated in the nuclear genome to form functional exon sequences. Taken together, our results shed light on the molecular features of NUMTs in the bumblebee nuclear genome and uncover their contribution to genome innovation.

Author Contributions: Conceptualization, C.S.; Methodology, L.D.; Software, L.D.; Resources, L.D. and H.S.; Data curation, H.S.; Writing — original draft preparation, C.S.; Writing—review and editing, H.S. All authors have read and agreed to the published version of the manuscript.

Funding: This research was funded by the Fundamental Research Funds of Chinese Academy of Agricultural Sciences (grant numbers: Y2019XK13 and Y2021XK16) and the Fundamental Research Funds for the Central Universities, grant number 2019FZJD007.

Institutional Review Board Statement: Not applicable.

Data Availability Statement: Mitogenomes generated in the study have been deposited in GenBank at the National Center for Biotechnology Information (NCBI), with accession numbers MZ352138-MZ352148.

Acknowledgments: The authors would like to thank Zhiqiang Wu and five reviewers for their constructive suggestions for improving the earlier versions of the manuscript.

Conflicts of Interest: The authors declare no conflict of interest.

\section{References}

1. Timmis, J.N.; Ayliffe, M.A.; Huang, C.Y.; Martin, W.F. Endosymbiotic gene transfer: Organelle genomes forge eukaryotic chromosomes. Nat. Rev. Genet. 2004, 5, 123-135. [CrossRef] [PubMed]

2. Kleine, T.; Maier, U.G.; Leister, D. DNA Transfer from Organelles to the Nucleus: The Idiosyncratic Genetics of Endosymbiosis. Annu. Rev. Plant Biol. 2009, 60, 115-138. [CrossRef] [PubMed]

3. Hazkani-Covo, E.; Zeller, R.M.; Martin, W. Molecular Poltergeists: Mitochondrial DNA Copies (numts) in Sequenced Nuclear Genomes. PLoS Genet. 2010, 6, e1000834. [CrossRef]

4. Ricchetti, M.; Fairhead, C.; Dujon, B. Mitochondrial DNA repairs double-strand breaks in yeast chromosomes. Nat. Cell Biol. 1999, 402, 96-100. [CrossRef] [PubMed]

5. Richly, E.; Leister, D. NUMTs in sequenced eukaryotic genomes. Mol. Biol. Evol. 2004, 21, 1081-1084. [CrossRef] 
6. Noutsos, C.; Richly, E.; Leister, D. Generation and evolutionary fate of insertions of organelle DNA in the nuclear genomes of flowering plants. Genome Res. 2005, 15, 616-628. [CrossRef]

7. Hazkani-Covo, E.; Covo, S. Numt-Mediated Double-Strand Break Repair Mitigates Deletions during Primate Genome Evolution. PLoS Genet. 2008, 4, e1000237. [CrossRef]

8. Michalovova, M.; Vyskot, B.; Kejnovsky, E. Analysis of plastid and mitochondrial DNA insertions in the nucleus (NUPTs and NUMTs) of six plant species: Size, relative age and chromosomal localization. Heredity 2013, 111, 314-320. [CrossRef]

9. Turner, C.; Killoran, C.; Thomas, N.S.T.; Rosenberg, M.; Chuzhanova, N.A.; Johnston, J.; Kemel, Y.; Cooper, D.N.; Biesecker, L.G. Human genetic disease caused by de novo mitochondrial-nuclear DNA transfer. Qual. Life Res. 2003, 112, 303-309. [CrossRef] [PubMed]

10. Leister, D. Origin, evolution and genetic effects of nuclear insertions of organelle DNA. Trends Genet. 2005, 21, 655-663. [CrossRef]

11. Noutsos, C.; Kleine, T.; Armbruster, U.; DalCorso, G.; Leister, D. Nuclear insertions of organellar DNA can create novel patches of functional exon sequences. Trends Genet. 2007, 23, 597-601. [CrossRef]

12. Zhang, D.-X.; Hewitt, G.M. Nuclear integrations: Challenges for mitochondrial DNA markers. Trends Ecol. Evol. 1996, 11, $247-251$. [CrossRef]

13. Williams, P. An Annotated Checklist of Bumble Bees with an Analysis of Patterns of Description (Hymenoptera: Apidae, Bombini). Bull. Nat. Hist. Mus. Entomol. 1998, 67, 79-152.

14. Cameron, S.A.; Hines, H.M.; Williams, P.H. A comprehensive phylogeny of the bumble bees (Bombus). Biol. J. Linn. Soc. 2007, 91, 161-188. [CrossRef]

15. Williams, P.H.; Cameron, S.A.; Hines, H.M.; Cederberg, B.; Rasmont, P. A simplified subgeneric classification of the bumblebees (genus Bombus). Apidologie 2008, 39, 46-74. [CrossRef]

16. Velthuis, H.; Doorn, A. A century of advances in bumblebee domestication and the economic and environmental aspects of its commercialization for pollination. Apidologie 2006, 37, 421-451. [CrossRef]

17. Goulson, D.; Lye, G.; Darvill, B. Decline and Conservation of Bumble Bees. Annu. Rev. Èntomol. 2008, 53, 191-208. [CrossRef]

18. Ricardo, P.C.; Françoso, E.; Arias, M.C. Mitochondrial DNA intra-individual variation in a bumblebee species: A challenge for evolutionary studies and molecular identification. Mitochondrion 2020, 53, 243-254. [CrossRef]

19. Sun, C.; Huang, J.; Wang, Y.; Zhao, X.; Su, L.; Thomas, G.W.C.; Zhao, M.; Zhang, X.; Jungreis, I.; Kellis, M.; et al. Genus-Wide Characterization of Bumblebee Genomes Provides Insights into Their Evolution and Variation in Ecological and Behavioral Traits. Mol. Biol. Evol. 2021, 38, 486-501. [CrossRef]

20. Sadd, B.M.; Barribeau, S.M.; Bloch, G.; De Graaf, D.C.; Dearden, P.; Elsik, C.G.; Gadau, J.; Grimmelikhuijzen, C.J.P.; Hasselmann, M.; Lozier, J.D.; et al. The genomes of two key bumblebee species with primitive eusocial organization. Genome Biol. 2015, 16, 76. [CrossRef]

21. Dierckxsens, N.; Mardulyn, P.; Smits, G. NOVOPlasty: De novo assembly of organelle genomes from whole genome data. Nucleic Acids Res. 2017, 45, e18. [PubMed]

22. Meng, G.; Li, Y.; Yang, C.; Liu, S. MitoZ: A toolkit for animal mitochondrial genome assembly, annotation and visualization. Nucleic Acids Res. 2019, 47, e63. [CrossRef]

23. Jin, J.-J.; Yu, W.-B.; Yang, J.-B.; Song, Y.; Depamphilis, C.W.; Yi, T.-S.; Li, D.-Z. GetOrganelle: A fast and versatile toolkit for accurate de novo assembly of organelle genomes. Genome Biol. 2020, 21, 241. [CrossRef] [PubMed]

24. Bernt, M.; Donath, A.; Jühling, F.; Externbrink, F.; Florentz, C.; Fritzsch, G.; Pütz, J.; Middendorf, M.; Stadler, P.F. MITOS: Improved de novo metazoan mitochondrial genome annotation. Mol. Phylogenetics Evol. 2013, 69, 313-319. [CrossRef]

25. Behura, S.K. Analysis of Nuclear Copies of Mitochondrial Sequences in Honeybee (Apis mellifera) Genome. Mol. Biol. Evol. 2007, 24, 1492-1505. [CrossRef] [PubMed]

26. Grabherr, M.G.; Haas, B.J.; Yassour, M.; Levin, J.Z.; Thompson, D.A.; Amit, I.; Adiconis, X.; Fan, L.; Raychowdhury, R.; Zeng, Q.; et al. Full-length transcriptome assembly from RNA-Seq data without a reference genome. Nat. Biotechnol. 2011, 29, 644-652. [CrossRef]

27. Cha, S.Y.; Yoon, H.J.; Lee, E.M.; Yoon, M.H.; Hwang, J.S.; Jin, B.R.; Han, Y.S.; Kim, I. The complete nucleotide sequence and gene organization of the mitochondrial genome of the bumblebee, Bombus ignitus (Hymenoptera: Apidae). Gene 2007, 392, 206-220. [CrossRef]

28. Du, Q.; Bi, G.; Zhao, E.; Yang, J.; Zhang, Z.; Liu, G. Complete mitochondrial genome of Bombus terrestris (Hymenoptera: Apidae). Mitochondrial DNA Part A 2015, 27, 4455-4456. [CrossRef]

29. Zhao, X.; Huang, J.; Sun, C.; An, J. Complete mitochondrial genome of Bombus consobrinus (Hymenoptera: Apidae). Mitochondrial DNA Part B 2017, 2, 770-772. [CrossRef]

30. Zhao, X.; Wu, Z.; Huang, J.; Liang, C.; An, J.; Sun, C. Complete mitochondrial genome of Bombus breviceps (Hymenoptera: Apidae). Mitochondrial DNA Part B 2017, 2, 604-606. [CrossRef]

31. Zhao, F.; Yan, J.; Jiang, K.; Huang, Z.; Lin, G. Nearly complete mitochondrial genomes of four bumblebee species (Hymenoptera: Apidae: Bombus). Mitochondrial DNA Part B 2019, 4, 183-184. [CrossRef]

32. Pamilo, P.; Viljakainen, L.; Vihavainen, A. Exceptionally High Density of NUMTs in the Honeybee Genome. Mol. Biol. Evol. 2007, 24, 1340-1346. [CrossRef] [PubMed] 
33. Lenglez, S.; Hermand, D.; Decottignies, A. Genome-wide mapping of nuclear mitochondrial DNA sequences links DNA replication origins to chromosomal double-strand break formation in Schizosaccharomyces pombe. Genome Res. 2010, 20, 1250-1261. [CrossRef]

34. Wang, J.-X.; Liu, J.; Miao, Y.-H.; Huang, D.-W.; Xiao, J.-H. Tracking the Distribution and Burst of Nuclear Mitochondrial DNA Sequences (NUMTs) in Fig Wasp Genomes. Insects 2020, 11, 680. [CrossRef] [PubMed]

35. Storchova, Z.; Breneman, A.; Cande, J.; Dunn, J.; Burbank, K.S.; O’Toole, E.; Pellman, D. Genome-wide genetic analysis of polyploidy in yeast. Nat. Cell Biol. 2006, 443, 541-547. [CrossRef] [PubMed] 